\title{
Thermal conductivity measurement in clay dominant consolidated material by Transient Hot-Wire method.
}

\author{
J. Ph. Garnier ${ }^{1, a}$, J. Gallier ${ }^{2}$, B. Mercx ${ }^{2}$, P. Dudoignon ${ }^{2}$ and D. Milcent ${ }^{3}$ \\ ${ }^{1}$ Laboratoire d'Etudes Thermiques, UMR CNRS 6608, Université de Poitiers - ESIP, \\ 40, Avenue du Recteur Pineau, 86022 Poitiers, France. \\ ${ }^{2}$ Laboratoire HYDRASA, Université de Poitiers, UMR 6269, Université de Poitiers - ESIP 40, \\ Avenue du Recteur Pineau, 86022 Poitiers, France. \\ ${ }^{3}$ Communauté de Commune du Lezayen, 79120 Lezay.
}

\begin{abstract}
The transient hot-wire (THW) technique is widely used for measurements of the thermal-conductivity of most fluids and some attempts have also been carried out for simultaneous measurements of the thermal-diffusivity with the same hot wire. This technique was also tried to determine thermal properties of soils by the mean of probes which can be considered as wire with some assumptions. The purpose of this paper is to validate the thermal conductivity measurement by the THW technique in geomaterials, composed of compacted sand + clay mineral that can be used for earth construction (Compacted Earth Brick). The thermal transfer behaviors are mainly governed by the texture and moisture of the geomaterials. Thus the investigations were performed (1) in media made of glass beads of different diameters in dry and saturated state in order to observe the role of grain sizes and saturation state on the wire temperature $(\Delta t)$ measurements and (2) in the compacted clay-geomaterial at different moisture states. The $\Delta \mathrm{t} / \ln (\mathrm{t})$ diagrams allow the calculation of two thermal conductivities. The first one, measured in the short time acquisition $(<1 \mathrm{~s})$, characterizes the microtexture of the material and its hydrated state. The second one, measured for longer time acquisitions, characterizes the mean thermal conductivity of the material.
\end{abstract}

\section{Introduction}

The methods of earth material building are commonly classed in «eco-construction » processes. In these objectives the earth materials have to demonstrate ecological qualities both in their production and building techniques but also on their thermal and hydric balances. The building techniques of earth material have been largely discussed and normalized. The material cohesion is assumed by the clay mineral matrix often added of some percents of lime or cement. Mechanical resistances are due to (1) the clay matrix cohesion which acts as cement between the sand grains and (2) compactness of the clay-sand assemblage which reduces the volume of microporosity by decreasing the micropore sizes. The clay matrix cohesion is governed by the initial water - clay ratio and compaction pressure applied during the earth material building $[1,2]$. The compactness of the material is also dependant

a email : jean.philippe.garnier@univ-poitiers.fr

This is an Open Access article distributed under the terms of the Creative Commons Attribution-Noncommercial License 3.0, which permits unrestricted use, distribution, and reproduction in any noncommercial medium, provided the original work is properly cited. 
of the textural continuity from the clayey to sandy components. All these parameters act on the fabrics of mineral particles and sand grains and, as a consequence, on the associated distribution of micro - to - meso porosity.

The heat transfers mainly depend on the frequency of grain contacts, size of pores and air - water ratio infilling the porosity. The moisture transfers mainly depend on the connectivity associated to the meso - to - microporosity distribution and especially on the microporosity of the clay matrix in clay dominant materials.

This work concerns the measurements of thermal conductivity in a clay dominant material composed of Compacted Earth Brick using the transient hot-wire (THW) technique. This hot-wire method has been developed for measurements in electro-conducting liquid [3-6] and in highly corrosive liquids [7-9]. Actually, the use of the transient hot-wire method is extended to granular materials [10,11], in solid geomaterials [12-15], and in soils by pedologists [16-19]. The texturemoisture and thermal conductivity relationship have been studied firstly on a model material composed of glass bead assemblages to demonstrate the role of porosity and saturation index. Secondly the thermal conductivities were measured on a compact earth brick mainly composed of clay material. In order to validate the THW method in this very thin divided material.

\section{Principle of the THW method}

The THW method is a transient dynamic technique based on the measurement of the temperature rising from a linear heat source (hot wire) embedded in the tested material [7, 8, 20]. For an infinitely long metallic wire (radius : $r_{0}$ ) heated at time $t>0$ with a constant heat flux per unit length $\mathrm{q}$ and immersed in an infinite homogeneous medium (thermal conductivity and diffusivity $: \lambda$ and a, respectively ) with uniform initial temperature, the rising wire temperature $\Delta \mathrm{T}(\mathrm{t})$ is given by [1]:

$$
\Delta T(t)=\left(\frac{q}{4 \cdot \pi \cdot \lambda}\right) \ln \left(\frac{4 \cdot F o}{C}\right)
$$

where $\gamma$ is the Euler's constant $\left(\gamma=0,5772\right.$.) with $\mathrm{C}=\mathrm{e}^{\gamma}=1,781$ et $\mathrm{F}_{\mathrm{O}}$ the Fourier number defined by :

$$
F o=\frac{a \cdot t}{r_{0}^{2}}
$$

Equation (1) is the analytical solution of an ideal thermal conductive model (only valid for Fo >> 1 and over a limited time interval, without convective transfers, in a liquid medium or not for a solid).

From this ideal model and with known q values, the thermal conductivity can be calculated by:

$$
\lambda=\left(\frac{q}{4 \cdot \pi}\right) \cdot\left(\frac{d T}{d(\ln t)}\right)^{-1}
$$

where $\mathrm{dT} / \mathrm{d}(\ell \mathrm{n} \mathrm{t})$ is a numerical constant deduced from experimental data for $\mathrm{t}$ values witch satisfy the condition Fo $>>1$.

For practical applications of the THW method, wire and material samples dimensions are finite, among other ideal model hypothesis. Then deviations from the ideal model have to be evaluated. In fact, the e (t) answer to the wire heating $\Delta \mathrm{T}(\mathrm{t})$ résultant of the Joule Effect due to an electrical current $i$ is :

$$
e(t)=R(t) \cdot i=R_{0}\left(1+\beta_{0} \cdot \Delta T(t)\right) i
$$


with $R(t)$ the instantaneous electrical resistance of the wire and $\beta_{0}$ the temperature coefficient of the wire $\left(R\left(T_{0}\right)=R_{0}\right)$. Taking into account the equations (3) and (4), the thermal conductivity $\lambda$ may be calculated as follows:

$$
\lambda=\left(\frac{q \cdot R_{0} \cdot \beta_{0} \cdot i}{4 \cdot \pi}\right) \cdot\left(\frac{d e(t)}{d(\ln t)}\right)^{-1}
$$

where $d e(t) / d(\ln (t))$ is a numerical constant deduced from the experimental data and from the linear part of the $\mathrm{e}(\mathrm{t})=\mathrm{f}(\ln (\mathrm{t}))$ curve.

\section{Materials and method}

The heat transfers were measured in assemblages of glass beads of different diameters and in sand + clay matrix compacted material (compact brick). The data (temperature versus time) were recording using a $\mathrm{Ta}$ or $\mathrm{Ni}$ wire of $0.125 \mathrm{~mm}$ diameter insert in the glass bead assemblages or in the compacted brick during its manufacturing. The heat transfers are induced by constant heating using $100 \mathrm{~mA}$ and $300 \mathrm{~mA}$ currents. The current generator has to be very precise. It is a Keithley 2400 (precision of $10^{-}$ ${ }^{6} \mathrm{~A}$ ). The nanovoltmeter used is a Keithley 2182 (precision of $10^{-10} \mathrm{~V}$ ). The experiments are driven by a microcomputer equipped with GPIB connections and Labview ${ }^{\circledR}$ software.

Seven glass bead media have been tested. They were characterized by bead diameters of: $0.50-$ 0.75, 0.75-1.0, 1.0-1.4, 1.7-2.1, 2.5-3, 2.8-3.4 and 3.9-4.4 mm. The Compacted Earth Brick is made by mechanical compression of earth plus sand mixture added of $5 \%$ lime. In weight percentage the composition of the compacted brick is $62 \%$ earth, $28 \%$ sand, $5 \%$ lime and $5 \%$ water. The mineralogy of sand is quartz dominant, feldspars plus accessory Fe oxides. The mineralogy of clayey fraction is Illite, Kaolinite and Smectite.

All the 7 glass beads media have been tested in air dry state. To underline the effect of saturation the 2.8-3.4 mm glass bead medium has been tested with water and acetone saturation. The thermal conductivity was measured in the compacted brick from initial $17 \%$ in weight of water content to $3 \%$ of residual water content after 110 days of air drying (Figure 1). The $17 \%$ of water content corresponds to a saturation index (Sat) of $90 \%$. The $3 \%$ correspond to a saturation index of $15 \%$. The measurements were made daily during the first sixty days and with larger steps ( $2-3$ days) after. The heat transfers measurements are represented in $\Delta \mathrm{T}\left({ }^{\circ} \mathrm{C}\right)$ versus $\ln (\mathrm{t})$ diagram (Figures 2 and 4). Each recorded $\Delta \mathrm{T} / \ln (\mathrm{t})$ curve presents two successive steps of temperature increasing characterized by $\mathrm{a}_{1}$ and $\mathrm{a}_{2}$ slopes which are used to calculate two associated heat conductivities $\lambda_{1}$ and $\lambda_{2}$.

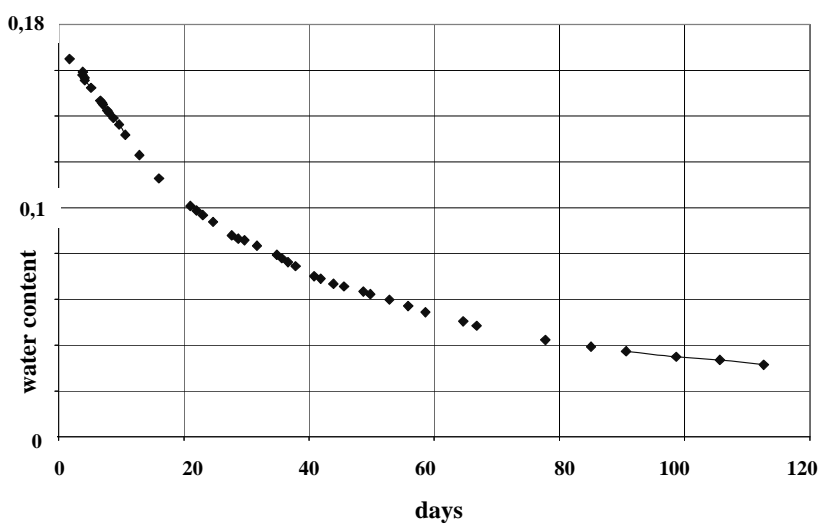

Fig. 1 Water content of the compact earth brick during the 110 days of air drying period. 


\section{Results and discussion}

For the glass bead materials all the $\Delta \mathrm{T} / \ln (\mathrm{t})$ curves show the same features :

-For $\mathrm{t}<1 \mathrm{~s}, \Delta \mathrm{T}$ increases quickly.

-For $\mathrm{t}>1 \mathrm{~s}, \Delta \mathrm{T}$ increases slowly.

These two domains are characterized by two $a_{1}$ and $a_{2}$ slopes respectively. In the short time domain $(t<1 \mathrm{~s})$ the $\mathrm{a}_{1}$ increases with the increasing of bead diameters. Actually the $\mathrm{a}_{1}$ slope is dependant of the density of beads contact with the wire. The associated $\lambda_{1}$ evolves from 0.11 to $0.038(\mathrm{~W} / \mathrm{mK})$. It can be expressed as a function of the bead diameter $(\mathrm{mm})$ as follows:

$$
\lambda_{1}=0.08 D^{-0.5}
$$

On the contrary, for the successive tests performed on the different material, the $\mathrm{a}_{2}$ slope is nearly constant independently of the bead sizes. According to the very low $a_{2}$ evolutions, the $\lambda_{2}$ is nearly constant and independent of the pore size for a constant porosity (about $0.2 \mathrm{~W} / \mathrm{mK}$ ).

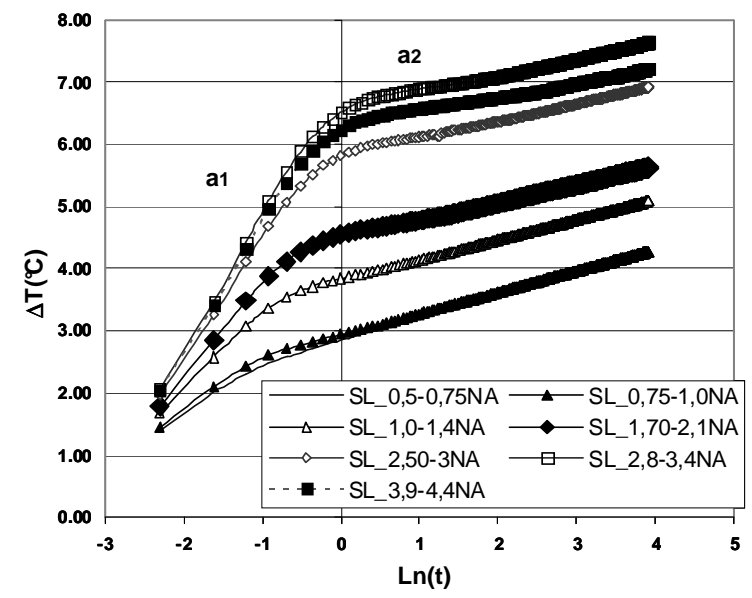

Fig. 2 Evolution of the slopes $\left(a_{1}, a_{2}\right)$ of the $\Delta \mathrm{T} / \ln (\mathrm{t})$ curves for the successive glass bead size materials $0.50-0.75,0.75-1.0,1.0-1.4,1.7-2.1,2.5-3,2.8-3.4$ and 3.9-4.4 mm. measurements with a $300 \mathrm{~mA}$ current. 


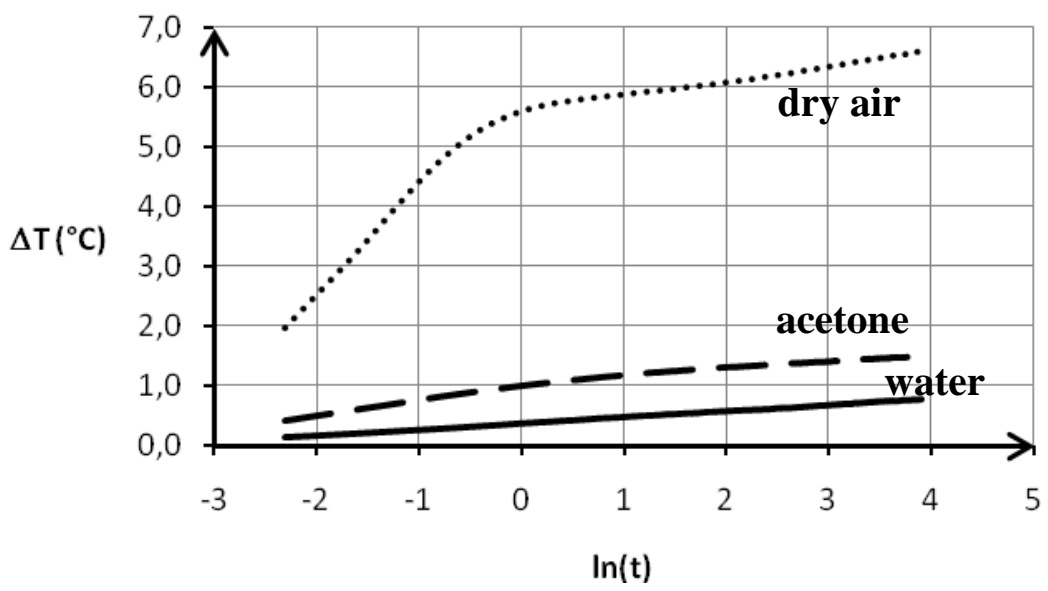

Fig. 3 Evolution the $\Delta \mathrm{T} / \ln (\mathrm{t})$ curves for 2.8-3.4 mm glass beads in dry air state, acetone and water saturated states. The Shift down of the $\Delta \mathrm{T} / \ln (\mathrm{t})$ curves curves due to the saturation accord with the decreasing thermal conductivities of the air $(\lambda=0.025 \mathrm{~W} / \mathrm{mK})$, acetone $(\lambda=0.19 \mathrm{~W} / \mathrm{mK})$ and pure water $(\lambda=0.6 \mathrm{~W} / \mathrm{mK})$.

The air/water or air/acetone substitution implies great decrease of the $\mathrm{a}_{1}$ slope on the $\Delta \mathrm{T} / \ln (\mathrm{t})$ curves and consequently a great decrease of $\lambda_{1}$ (Figure 3 ). The $a_{2}$ slope and $\lambda_{2}$ show only weak evolutions.

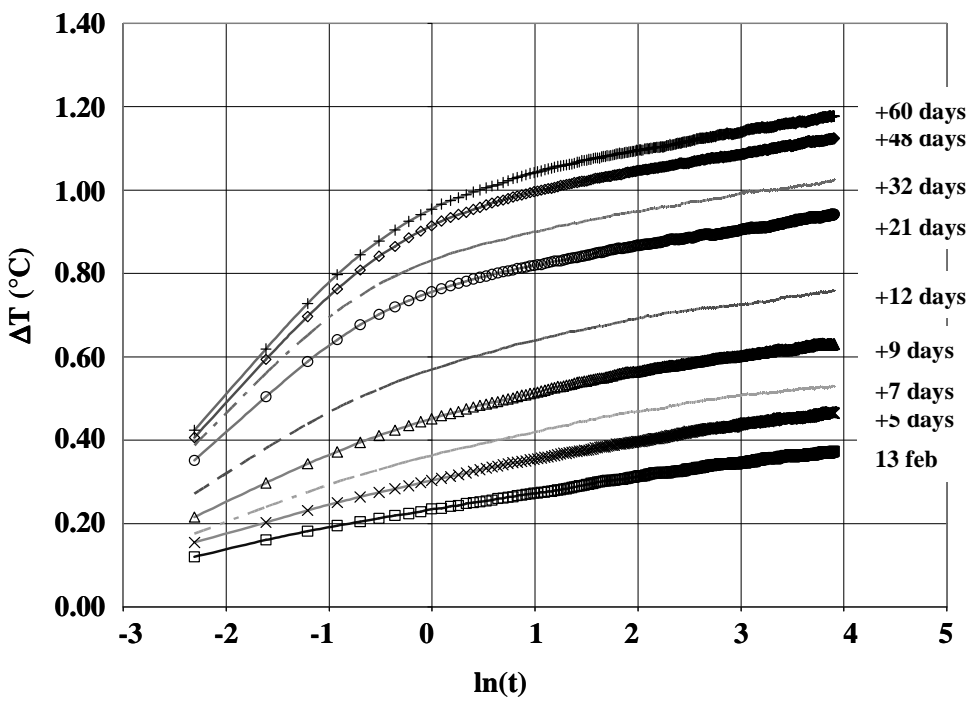

Fig. 4 Evolution of the $\Delta \mathrm{T} / \ln (\mathrm{t})$ curves recorded on the compacted brick with time from 13 February (Fev) to April (+ 60 days). The shift of the curves from the abscissa to the top of the diagram is due to the desiccation of the clay material. The consequence is the drastic evolution of the a1 slope.

For the compacted earth brick, the $\Delta \mathrm{T} / \ln (\mathrm{t})$ curves show similar features :

- For $\mathrm{t}<1 \mathrm{~s}, \Delta \mathrm{T}$ increases quickly.

- For $\mathrm{t}>1 \mathrm{~s}, \Delta \mathrm{T}$ increases slowly. 
For this material, only one brick was tested, thus only one texture was tested. The evolution of the thermal conductivity is only dependant of the evolution of the brick water content. In these conditions the evolutions of $\mathrm{a}_{1}$ and $\mathrm{a}_{2}$ during the drying period characterize the decrease of moisture. The first $\Delta \mathrm{T} / \ln (\mathrm{t})$ curves recorded during the first week show quite flat patterns. On the contrary the curves recorded at the end of the drying period (110 days) shown clearly the two different $\mathrm{a}_{1}$ and $\mathrm{a}_{2}$ slopes (Figure 4). The first curves were recorded for saturation index near $20 \%$ and the last measurement series were performed for saturation index of $90 \%$. The evolution of $\Delta \mathrm{T} / \ln (\mathrm{t})$ curve patterns and slopes $a_{1}$ and $a_{2}$ have consequences on the calculated $\lambda_{1}$ an $\lambda_{2}$ (Figure 5): weak $\lambda_{2}$ evolution with time but drastic decease of $\lambda_{1}$ with the saturation index decrease in the material.

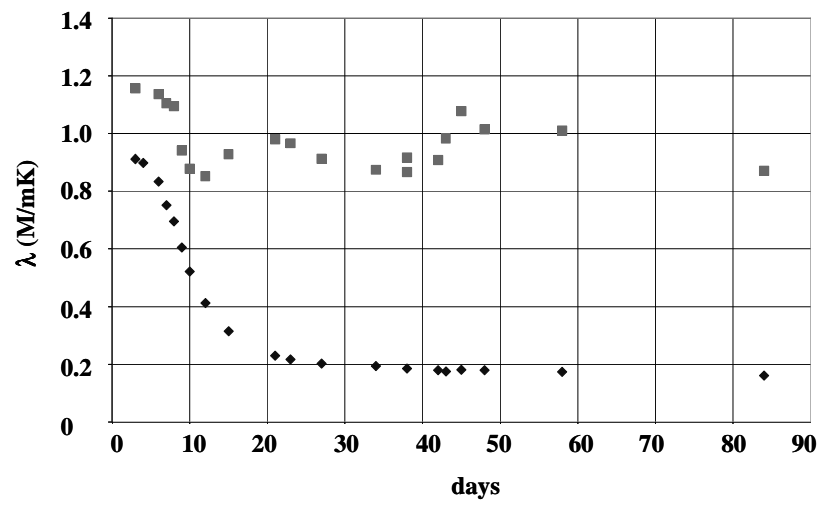

Fig. 5 Evolution of $\lambda_{1}$ (lozanges) an $\lambda_{2}$ (squares) thermal conductivity with time. According to the drastic a1 increase the $\lambda_{1}$ values decrease from 0.9 to $0.2 \mathrm{M} / \mathrm{mK}$ during the 20 firts days. The $\lambda_{2}$ show high dispesive values for a mean value quite constant with time..

The microstructre of the compacted brick was observed on scanning electronic microscope. The clay matrix is very compact. All the porosity is constituted of microporosity disseminated between the clay particles and almost all the micropores have sizes lower than $20 \mu \mathrm{m}$ (Figure 6). Nevetheless the $\Delta \mathrm{T} / \ln (\mathrm{t})$ curves show similar pattern evolutions between saturated and unsaturated states than glass bead ones.

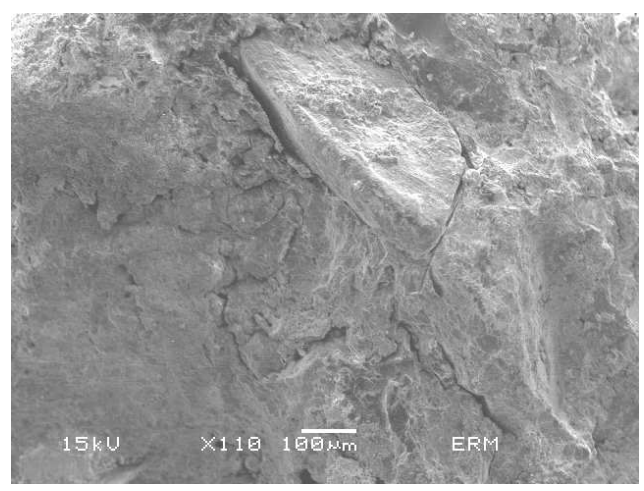

Fig. 6 SEM micro-photography of the microstructure of the compacted brick. The sand grain is isolated inside the compacted clay matrix. The microcracks are consequences of the sampling of the milimetric block from the brick. The clay matrix appears very compact and homogeneous. 


\section{Conclusion}

The method of thermal conductivity measurement by the Hot Wire Method, already validated in fluids appears to be well adapted to thermal conductivity measurement in solid and clay dominant material. The experiments on glass beads show interesting correlations between the $\Delta \mathrm{T} / \ln (\mathrm{t})$ curves patterns and (1) the microstructure of the material and (2) saturation state of the material. The measurements of the clay dominant material show similar results instead of the very thin size of particles and associated microporosity. The $\lambda 1$ coefficient obtain in short time (around $1 \mathrm{~s}$ ) is sufficient to characterize the moisture and saturation index of the material for one know grain size distribution (Figure 1). As a conclusion, the Hot Wire Method developed to the thermal conductivity measurement in liquid is well adapted to similar measurements in solid materials, besides the method is able to give characteristics on the size pores in geomaterials and saturation index of the medium.

These preliminary results suggest the possibility of investigation on the microstructure and associated water content behavior of geomaterials. The technique may be extended to in situ measurements in wall, soils, and other materials in order to record data of thermo-hydric transfers and to model the mechanisms.

\section{References}

1. P. Dudoignon, D. Gélard,, S. Sammartino. Cam-clay and hydraulic conductivity diagram relations in consolidated and sheared clay-matrices. Clay Minerals, 269-279. 13 (2004)

2. D. Gélard, and P. Dudoignon, Mécanisme de réorganisation de matrice argileuse par séchage dans un matériau terre : influence sur la cohésion AUGC (2007)

3. Y. Nagasaka and A. Nagashima Absolute Measurement of the Thermal Conductivity of Electrically Conducting Liquids by the Transient Hot-Wire Method J. Phys. E: Sci. Instrum, 1435144014 (1981)

4. J. R. Parsons, Jr. and J. C. Mulligan Measurement of the Properties of Liquids and Gases using a Transient Hot-Wire Technique Rev. Sci. Instrum., 1460-1463 49 (1978)

5. W. Alloush, B. Gosney, and W. A. Wakeham. A Transient Hot-Wire Instrument for Thermal Conductivity Measurements in Electrically Conducting Liquids at Elevated Temperatures International Journal of Thermophysics, 225-235 3 (1982)

6. M. L. V. Ramires, J. M. N. A. Fareleira, C. A. Nieto de Castro, The Thermal Conductivity of Toluene and Water International Journal of Thermophysics, 1119-1130 14 (1993)

7. J.P. Garnier, J.P. Maye, J. Saillard, G. Thévenot, A. Kadjo, S. Martemianov. A New Transient Hot-Wire Instrument for Measuring the Thermal-Conductivity of Electrically Conducting and Highly Corrosive Liquids using Small Samples, International Journal of Thermophysics, 468-482 29 (2008)

8. A. Kadjo, J.P. Garnier, J.P. Maye, S. Martemianov A New Transient Two-Wire Method for Measuring the Thermal Diffusivity of Electrically Conducting and Highly Corrosive Liquids Using Small Samples International Journal of Thermophysics, 1267-1277 29 (2008)

9. G. Thevenot, J. Saillard, J.-P. Maye, J.-Ph. Garnier. Cellule de mesure de la conductivité et de la diffusivité thermiques d'un fluide et sonde pour cette cellule European Patent Office. EP 1724572 A1 (2006)

10. E. Gonzo, Estimating correlations for the effective thermal conductivity of granular materials Chemical Engineering Journal, 299-302 90 (2002)

11. G.K. Batchelor, R.W. O'Brien, thermal or electrical conduction through a granular material, Proc.R.Soc.Lond, 313-333 355 (1977)

12. C. Gruescu, A. Giraud , F. Homand, D. Kondo, D.P. Do Effective thermal conductivity of partially saturated porous rocks International Journal of Solids and Structures, 811-833 44 (2007) 
13. A. Bouguerra, A. Ledhem, F. de Barquin, R.M. Dheilly, and M. Quéneudec Effect of microstructure on the mechanical and thermal properties of lightweight concrete prepared from clay, cement and wood aggregates Cement and Concrete Research 1179-1190 28 (1998)

14. H.Ezbakhe, Caractérisation thermiques et mécaniques des matériaux poreux utilisés comme isolants simples ou porteurs, Thèse de doctorat, UCPB Lyon 1 (1986).

15. J.P.Cull, thermal conductivity probes for rapid measurement in rock. J.Phys.E: Sci.Instrum, 7717747 (1974)

16. K.H. Bristow Measurement of thermal properties and water content of unsaturated sandy soil using dual-probe heat pulse probes Agricultural and forest meteorology, 75-84 89 (1998)

17. K.H. Bristow, G.J. Kluitenberg, C.J. Golding, T.S. Fitzgerald A small multi-needle probe for measuring soil thermal properties, water content and electrical conductivity Computers and Electronics in Agriculture, 265-280 31 (2001)

18. K.H. Bristow, Measurement of thermal properties and water content of unsaturated sandy soil using dual-probe heat pulse probes Agricultural and forest meteorology, 75-84 89 (1998)

19. D.A DE. Vries, A.J.Peck, On cylindrical probe method of measuring thermal conductivity with special reference to soils, Aust.J.Phys 225-271 11 (1958)

20. J. J. Healy, J. J. De Groot and J. Kestin The Theory of the Transient Hot-Wire Method for Measuring Thermal Conductivity Physica 392-408 82C (1976) 